SLAC-PUB-10693

September 2004

\title{
Baryonic States in QCD From Gauge/String Duality at Large $\mathbf{N}_{\mathbf{C}^{*}}$
}

\author{
Guy F. de Téramond ${ }^{\dagger}$ and Stanley J. Brodsky ${ }^{\ddagger}$ \\ Universidad de Costa Rica, San José, Costa Rica \\ Stanford Linear Accelerator Center, \\ Stanford University, Stanford, California 94309, USA
}

\begin{abstract}
We have computed the baryon spectrum in the framework of $\mathcal{N}=4$ superconformal Yang-Mills theory using AdS/CFT duality. Baryons are included in the theory by adding an open string sector, corresponding to quarks in the fundamental representation of $\mathcal{N}=4$. The hadron mass scale is introduced by imposing boundary conditions at the $A d S_{5}$ coordinate $r_{0}=\Lambda_{Q C D} R^{2}$, which is the only parameter. The quantum numbers of each baryon, are identified by matching the fall-off of the string wavefunction $\Psi(x, r)$ at the asymptotic $3+1$ boundary to the operator dimension of the lowest three-quark Fock state, subject to appropriate boundary conditions. Higher Fock states are matched quanta to quanta with quantum fluctuations of the bulk geometry about the AdS background. We limit our discussion to massless quarks. The resulting four-dimensional spectrum displays a remarkable resemblance to the physical baryon spectrum of QCD, including the suppression of spin-orbit interactions.
\end{abstract}

Presented at
Workshop on Large $N_{C}$ QCD 2004
5-9 July 2004
Trento, Italy

${ }^{*}$ Work supported by the Department of Energy contract DE-AC02-76SF00515.

${ }^{\dagger}$ E-mail: gdt@asterix.crnet.cr

${ }_{\ddagger}^{\ddagger}$ E-mail: sjbth@slac.stanford.edu 


\section{Introduction}

An outstanding consequence of Maldacena's duality [1] between 10-dimensional string theory on $A d S_{5} \times S^{5}$ and Yang-Mills theories at its conformal 3+1 spacetime boundary $[2,3]$ is the potential to describe processes for physical QCD which are valid at strong coupling and do not rely on perturbation theory. As shown by Polchinski and Strassler [4], dimensional counting rules [5, 6] for the leading power-law fall-off of hard exclusive glueball scattering can be derived from a gauge theory with a mass gap dual to supergravity in warped spacetimes. The modified theory generates the hard behavior expected from QCD, instead of the soft behavior characteristic of strings. Other examples are the description of deep inelastic scattering [7] and the derivation of a heavy quark potential [8]. The discussion of scaling laws in warped backgrounds has also been addressed in $[9,10,11]$.

The AdS/CFT duality gives a non-perturbative definition of quantum gravity in a curved background which is asymptotic to a product of Anti-de Sitter space AdS and a compact Einstein space $X$. As originally formulated [1], a correspondence was established between the supergravity approximation to Type IIB string theory, and the large $N_{C}$ brane decoupling limit with gauge dynamics corresponding to $\mathcal{N}=4$ super Yang-Mills (SYM) in four dimensions. The bulk geometry has exact conformal geometry $A d S_{5} \times S^{5}$ in the near-horizon region $r \ll R$, where $R=\left(4 \pi g_{s} N_{C}\right)^{1 / 4} \alpha^{1 / 2}$ is the radius of AdS and the radius of the five-sphere. The extra five dimensions of $S^{5}$ correspond to the $S U(4)$ global symmetry which rotates the particles present in the SYM supermultiplet in the adjoint representation of $S U\left(N_{C}\right)$. The conformal group $S O(2,4)$ is identified with the isometry group of $A d S_{5}$, and $S U(4) \simeq S O(6)$ with the isometries of $S^{5}$. The supergravity duality requires a large value of $R$ corresponding to a large value of the 't Hooft parameter, $g_{s} N_{C}$ [12].

In a recent attempt to extend the glueball results to hadrons [13], we used the AdS/CFT correspondence to determine the basic properties of hadronic light-front wavefunctions (LFWF) in QCD based on the underlying conformal symmetry of the duality for pointlike hard-scattering processes which occur in the large- $r$ region of AdS space. The scaling behavior of the string modes determine the behavior of the QCD hadronic wavefunction, giving a precise counting rule for each Fock component state with an arbitrary number of quarks and gluons and internal orbital angular momentum ${ }^{1}$. The discussion was carried out in terms of the lowest dimensions of interpolating fields near the boundary of AdS, treating the boundary values of the string states $\Psi(x, r)$ as a product of quantized operators which create $n$-partonic states out of the vacuum [13]. Our AdS/CFT derivation validate QCD perturbative results and confirm the dominance of the quark interchange mechanism [14] for exclusive

\footnotetext{
${ }^{1}$ In [13] we examined the possibility of identifying the internal orbital momentum of hadrons with Kaluza-Klein excitations of the internal space $S^{5}$. Henceforth we follow the interpretation given here in terms of quantum fluctuations.
} 
QCD processes at large $N_{C}$. The predicted orbital dependence coincides with the falloff of light-front Fock wavefunctions derived in perturbative QCD [15]. Since all of the Fock states of the LFWF beyond the valence state are a manifestation of quantum fluctuations, it is natural to match quanta to quanta the additional dimensions with the metric fluctuations of the bulk geometry about the fixed AdS background.

For large values of $R$, or small curvature of AdS space, it is expected that the dual of a Yang Mills theory is classical gravity. The correspondence also implies that the dual of strongly coupled QCD is a weakly coupled string. Since QCD is weakly coupled at high energies, the dual theory is expected to be a strongly coupled string model at small 't Hooft coupling and would require the understanding of strings in highly curved backgrounds, extending the semiclassical approximation to include quantum effects on the string theory side ${ }^{2}$. The behavior of string states in the infrared region is dependent on dynamics at small- $r$, and it is a priori unknown. Non-conformal aspects are needed to make contact with the real world. The introduction of quarks in the fundamental representation is also crucial, requiring an open string sector.

In spite of the difficulties mentioned above, important progress has been achieved by extending the AdS/CFT correspondence beyond the supergravity approximation to construct string duals to non-conformal gauge theories ${ }^{3}$. Even if the detailed form of the metric at small-r is unknown, salient QCD dynamical features, such as the generation of a mass gap and a hadron spectrum, will follow from the deformation of the AdS conformal background at small $r$. Indeed from [4] there follows a simple relation between the 10-dimensional string scale $\alpha_{s}^{\prime}$ and the Yang-Mills 4-dimensional scale $\alpha_{Q C D}^{\prime}$ in a warped space: $\alpha_{Q C D}^{\prime} \sim \alpha_{s}^{\prime}\left(R / r_{o}\right)^{2}$, where the cutoff $r_{o}=\Lambda_{Q C D} R^{2}$, breaks conformal invariance and allows the introduction of the QCD scale.

A physical hadron in four-dimensional Minkowski space has four-momentum $P_{\mu}$ and hadronic invariant mass states given by $P_{\mu} P^{\mu}=\mathcal{M}^{2}$. The string wavefunction in $r$ is the extension of the baryon wavefunction into the fifth dimension: we thus analytically match the three-quark proton wavefunction in $3+1$ space to its corresponding string wavefunction using the 10-dimensional Dirac equation. The physical string modes are plane waves along the Poincaré coordinates, which are coupled to the matter fields of the conformal theory as determined by the boundary limit between the string partition function and the generating functional of the quantum field theory $[2,3]$. For example, the quantum numbers of each baryon are determined by matching the dimensions of the string modes $\Psi(x, r)$, with the lowest dimension of the baryonic interpolating operators in the conformal limit. The specific correspondence gives us a prescription to map string modes into boundary states with well defined number of partons.

After stating in Sec. 2 some basic properties of the correspondence between string

\footnotetext{
${ }^{2}$ In the limit of large angular momentum on $S^{5}$, the strings can be quantized exactly in a plane pp-wave metric as a product of oscillatory excitations. For a review see [16].

${ }^{3}$ For a recent review see [17]. See also [12].
} 
modes in AdS space and baryon states at the asymptotic boundary, we confront our results with the spectrum of the known nucleon and $\Delta$ resonances in Sec. 3. We also examine the possibility that Roper like states would correspond to states present in the $S^{5}$ sector of the baryon spectrum. Some concluding remarks are given in Sec. 4 .

\section{Baryon Interpolating Operators and String Modes}

A precise statement of the duality between a string/gravity theory on a $(d+1)$ dimensional Anti-de Sitter space $A d S_{d+1}$ and the large $N_{C}$ limit of a conformal theory at its $d$-dimensional boundary, is given formally in terms of the full partition function of the string theory in the bulk $Z_{\text {string }}$ which should coincide with the generating functional of the conformal field theory $Z_{C F T}$ on the AdS boundary $[2,3]$ : $Z_{\text {string }}[\Psi(x, z=0)]=Z_{C F T}\left[\Psi_{o}\right]$. For spin- $\frac{1}{2}$ dilatino in the bulk, the duality involves positive and negative chirality components [18] which couple with CFT operators $\mathcal{O}^{+}$ and $\mathcal{O}^{-}$

$$
Z_{C F T}\left[\Psi_{o}^{ \pm}\right]=\left\langle\exp \left(i \int d^{d} x\left[\overline{\mathcal{O}}^{+} \Psi_{o}^{+}+\bar{\Psi}_{o}^{-} \mathcal{O}^{-}\right]\right)\right\rangle .
$$

Near the boundary of AdS, $z=R^{2} / r \rightarrow 0$, the independent solutions of the 10dimensional Dirac equation are

$$
\Psi^{ \pm}(z, x) \rightarrow z^{\frac{d}{2}-m R} \Psi_{-}^{ \pm}(x)+z^{\frac{d}{2}+m R} \Psi_{+}^{ \pm}(x) .
$$

The solution with $\Psi_{-}$dominates near $z \rightarrow 0$, thus $\Psi_{-}=\Psi_{o}$. The field $\Psi_{-}$acts as a boundary source, and $\Psi_{+}(x)$ is the response function which incorporates the quantum fluctuations. The boundary sources for positive and negative chirality $\Psi_{o}^{+}$ and $\Psi_{o}^{-}$have dimensions $\frac{d}{2}-m R$. Consequently the dimension of the CFT operators $\mathcal{O}^{+}$and $\mathcal{O}^{-}$is $\frac{d}{2}+m R$. Since the dimension of $\Psi_{+}$is also $\frac{d}{2}+m R$, we expect that $\Psi_{+}(x)$ is related to the expectation value of $\mathcal{O}$ in the presence of the source $\Psi_{o}$ : $\Psi_{+}(x) \sim\langle\mathcal{O}\rangle_{\Psi_{o}}$. Indeed $\langle\mathcal{O}\rangle=(2 \Delta-d) \Psi_{+}(x) \equiv \Psi_{\mathrm{cl}}(x)[19]$. Thus $\Psi_{+}$acts as a semiclassical field $\Psi_{\mathrm{cl}}$ and is the boundary limit of the normalizable string solution: $\Psi_{\mathrm{cl}}(x)=\langle\Omega|\mathcal{O}(x)| \Omega\rangle_{\Psi_{o}}$.

We consider first the classical solution dual to the valence Fock state, described by the massless 10-dimensional Dirac equation in the bulk: $\Gamma^{A} D_{A} \hat{\Psi}=0$. Full space coordinates are $x^{A}=\left(x^{\mu}, z, y^{a}\right)$, with $x^{\mu}$ the Minkowski variables and $z$ the holographic coordinate. Coordinates in the compact space $X$ are $y^{a}$. A 10-dimensional field is represented by hat quantities: $\hat{\Phi}, \hat{\Psi}$; a field on $\operatorname{AdS}$ space by $\Phi, \Psi$, and $\phi, \psi$ represent fields in 4-dimensions. Knowledge of the full geometry is required to solve the Dirac equation. Since we expect AdS metrics for $r \gg r_{o}$, a conformal solution with a cutoff at $r_{o}=\Lambda_{Q C D} R^{2}$ should describe to a good approximation the physics 
at small and large-r. We expand the state $\hat{\Psi}$ in terms of spinors $\eta(y)$ of the Dirac operator on a $d+1$ sphere with eigenvalues $\lambda_{\kappa}$ as $\hat{\Psi}(x, z, y)=\sum_{\kappa} \Psi_{\kappa}(x, z) \eta_{\kappa}(y)$. For each eigenvalue $\lambda_{\kappa}$ the normalizable string modes are

$$
\Psi_{\kappa}(x, z)=C e^{-i P \cdot x} z^{\frac{d+1}{2}}\left[J_{\alpha_{\kappa}}(z \mathcal{M}) \mu_{+}(P)+J_{\alpha_{\kappa}+1}(z \mathcal{M}) \mu_{-}(P)\right],
$$

where $\alpha_{\kappa}=\lambda_{\kappa} R-\frac{1}{2}$. Four dimensional spinors are related by $\mu_{-}=\hat{P} \mu_{+}, \hat{P}_{\mu}=$ $P_{\mu} /|P|$. The eigenvalues on $S^{d+1}$ are $\lambda_{\kappa} R= \pm\left(\kappa+\frac{d}{2}+\frac{1}{2}\right), \kappa=0,1,2, \ldots$, with multiplicity $[20] D_{d+1}(\kappa)=2^{\frac{d}{2}}\left(\begin{array}{c}d+\kappa \\ \kappa\end{array}\right)$.

The lowest dimension of a spin- $\frac{1}{2}$ field on $A d S_{d+1}$ space is $\operatorname{Dim}[\Psi]=d+\frac{1}{2}$. A spin- $\frac{1}{2}$ field in the d-dimensional boundary theory has dimensions $\operatorname{Dim}[\psi]=(d-1) / 2$. It is quite remarkable that a dual CFT boundary operator $\mathcal{O}$ with dimension $d+\frac{1}{2}$ can only be constructed for $\mathrm{d}=2,4$ and $\mathrm{d} \rightarrow \infty$ with a product of five, three and two quark fields respectively, to match the string dimensions in the bulk.

For $d=4$, the spinor irreps are 4, 20, 60, 140, . Classical spin- $\frac{1}{2}$ string solutions are labeled by eigenvalues of the Dirac operator on $X$. The lowest string mode for $\kappa=0$ has dimension $\Delta=\frac{9}{2}$, and transforms as a 4 under the $S U(4) R$-symmetry. The corresponding CFT operator $\mathcal{O}_{\frac{9}{2}}$ is constructed as the product of three quark fields $\psi$ transforming as a $\overline{\mathbf{4}}$ of $S U(4)$, since $\overline{\mathbf{4}} \otimes \overline{\mathbf{4}} \otimes \overline{\mathbf{4}} \rightarrow \mathbf{4}$. With respect to color $\mathcal{O}_{9 / 2}$ has the gauge invariant form $\mathcal{O}(x)_{9 / 2}=\psi_{\mathbf{N}_{\mathbf{C}}}(x) \psi_{\mathbf{N}_{\mathbf{C}}}(x) \psi_{\mathbf{N}_{\mathbf{C}}\left(\mathbf{N}_{\mathbf{C}}-\mathbf{1}\right) / \mathbf{2}}(x)$, where the representation $\mathbf{N}_{\mathbf{C}}\left(\mathbf{N}_{\mathbf{C}}-\mathbf{1}\right) / \mathbf{2}$ follows from the antisymmetric component of the tensor product $\mathbf{N}_{\mathbf{C}} \otimes \mathbf{N}_{\mathbf{C}}$. For $N_{C}=3$, we recover the usual form of the interpolating operator which creates a physical baryon in $\operatorname{QCD}(3+1): \mathcal{O}_{9 / 2}=\epsilon_{a b c} \psi_{a} \psi_{b} \psi_{c}$.

QCD is fundamentally different from SYM theories where matter fields appear in adjoint multiplets of $S U\left(N_{C}\right)$. The $\mathcal{N}=4$ theory is dual to the low energy supergravity approximation to type IIB string [1] compactified on $A d S_{5} \times S^{5}$. The SYM fields correspond to closed strings and comprise a gluon field $A_{\mu}$, six scalars $\phi_{I}, I=1, \ldots, 6$, four Majorana gluinos $\chi_{r}, r=1, \ldots, 4$, and their antiparticles $\chi^{r}$. The introduction of quarks in the fundamental representation is dual to the introduction of an open string sector, where the strings end on a brane and join together at a point in the AdS geometry [21]. Since the quarks fields $\psi^{r}$ transform as a $\overline{4}$ under $S U(4)$, the new degeneracy could be interpreted as a flavor symmetry for $r=u, d, c, s$, which is broken by quark masses ${ }^{4}$.

The AdS/CFT correspondence is interpreted in the present context as a classical duality between the lowest three-quark valence state in the asymptotic $3+1$ boundary and the lowest string mode in $A d S_{5} \times S^{5}$. Higher Fock components are a manifestation of the quantum fluctuations of QCD and are conformal states in the limit of massless quarks and vanishing QCD $\beta$-function. Metric fluctuations of the bulk geometry about the fixed AdS background should correspond to quantum fluctuations

\footnotetext{
${ }^{4}$ The introduction of a finite number of $N_{f}$ branes is dual to the introduction of flavor [22], and leads to a calculable spectrum [23, 24].
} 
of Fock states above the valence state in the limit where QCD appears nearly conformal [25]. We assume that the conformal dimensions of fluctuations of the underlying string theory are determined in the weakly coupled string approximation by matching dimensions with the physical hadronic interpolating operators at the Minkowski boundary, while maintaining conformal invariance.

A gauge invariant interpolating boundary operator has the generic form

$$
\mathcal{O}_{n}^{L}=\operatorname{Tr}\left(\phi \phi \ldots \psi D^{L} \psi \psi \ldots \bar{\psi} \psi \ldots F \ldots\right)
$$

with dimension $\Delta=\Delta_{n}+L$, where $\Delta_{n}$ is the sum of the dimensions of the quarks, antiquarks, vector and scalar gluons of a given Fock state, and $\mathrm{L}$ is the orbital momentum. In the conformal limit an $l$-quantum in the boundary corresponds to a mass $\mu \sim l / R$ in the string side. The interpolating operator with orbital $L$ couples to an $L=l$ quantum fluctuation of the AdS geometry. To describe an arbitrary Fock-state, we make the substitution $\alpha \rightarrow \alpha_{n}^{L}=d / 2+L+\Delta_{n}-\Delta_{v}$ in (3), where $\Delta_{v}$ is the dimension of the valence state. Mass eigenvalues are determined by imposing boundary conditions on one of the solutions of the Dirac equation $\Psi^{ \pm}\left(x, z=z_{o}\right)=0$. The solution of the spin- $\frac{3}{2}$ Rarita-Schwinger equation in AdS space is more involved, but considerable simplification occurs for polarization along Minkowski coordinates, $\Psi_{\mu}$, where it becomes similar to the spin- $\frac{1}{2}$ solution [26].

\section{Baryon Spectrum}

The study of the hadron spectrum is crucial for our understanding of bound states of strongly interacting relativistic confined particles. Different QCD-based models often disagree, even in the identification of the relevant degrees of freedom [27, 28]. Studies of orbitally excited baryons based on the $1 / N_{C}$ expansion have been useful for identifying the relevant effective operators and determine their relative importance [29]. An outstanding puzzle is that the spin-orbit splitting, which experimentally is very small, appear in the $1 / N_{C}$ expansion as a zeroth-order effect. Very recently, the computation of orbital and radial excitations on the lattice has been extended up to spin- $\frac{5}{2}$ states [30].

The known $N$ and $\Delta$ baryon states are listed in Table 1 according to total angular momentum-parity assignment given by the PDG [31]. To determine intrinsic spin and orbital momentum quantum numbers we have used the conventional $S U(6) \supset$ $S U(3)_{\text {flavor }} \otimes S U(2)_{\text {spin }}$ multiplet structure. A possible assignment of $S^{5}$ charges is also indicated. We limit ourselves to the light unflavored hadron states and the introduction of massless quarks. Since $m_{u, d} \ll \Lambda_{Q C D}$, the light quarks are extremely relativistic. Consequently the mass of the hadrons corresponds essentially to the confined kinetic energy of massless quarks and gluons. The intrinsic spin $S$ of a given hadron should match the spin of the dual string in the bulk. 


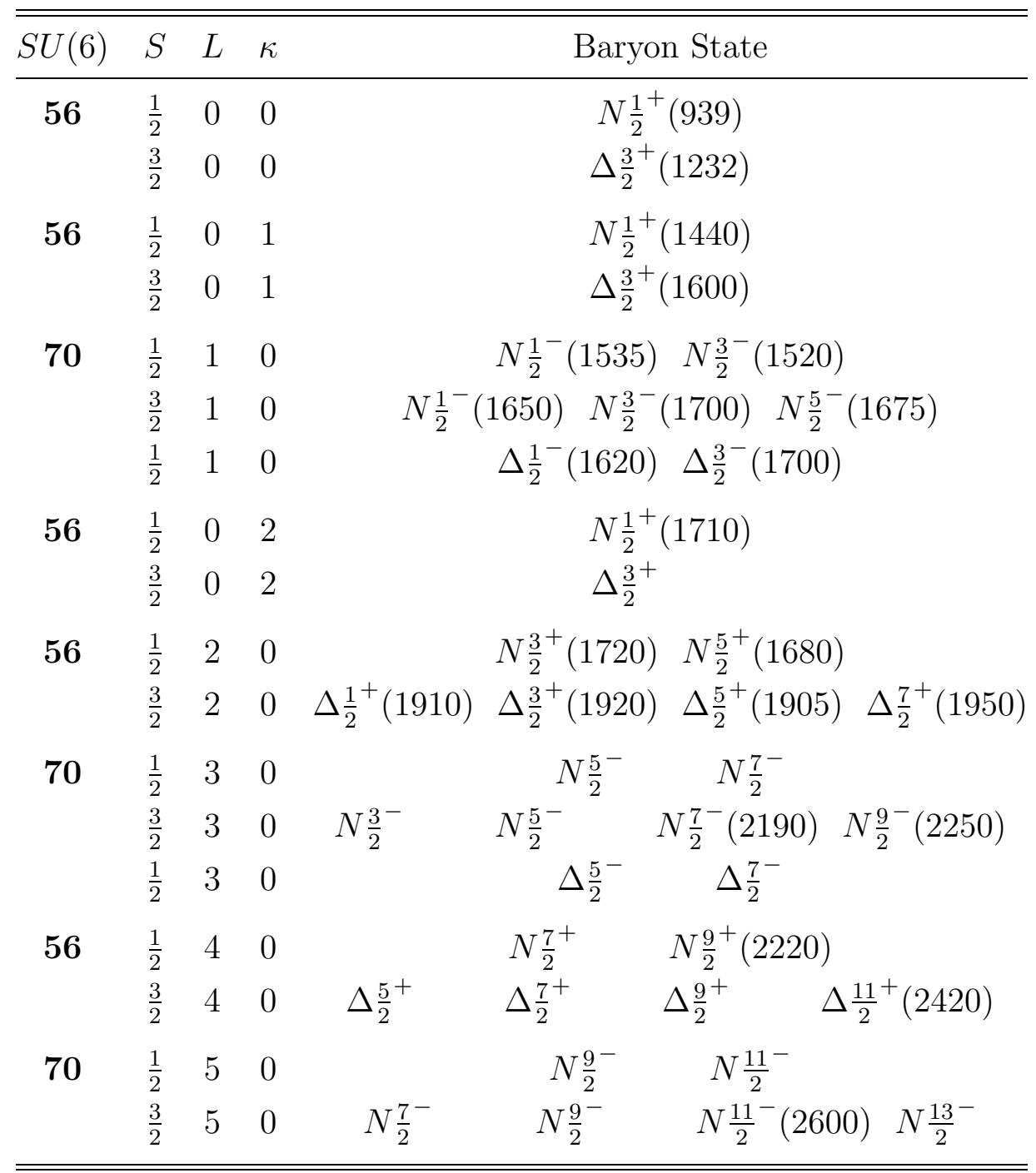

Table 1: $S U(6)$ multiplet structure for the known $N$ and $\Delta$ baryon resonances including internal spin $S$ and orbital angular momentum $L$ quantum numbers. A possible assignment of $S^{5}$ baryonic charge $\kappa$ is also indicated. 


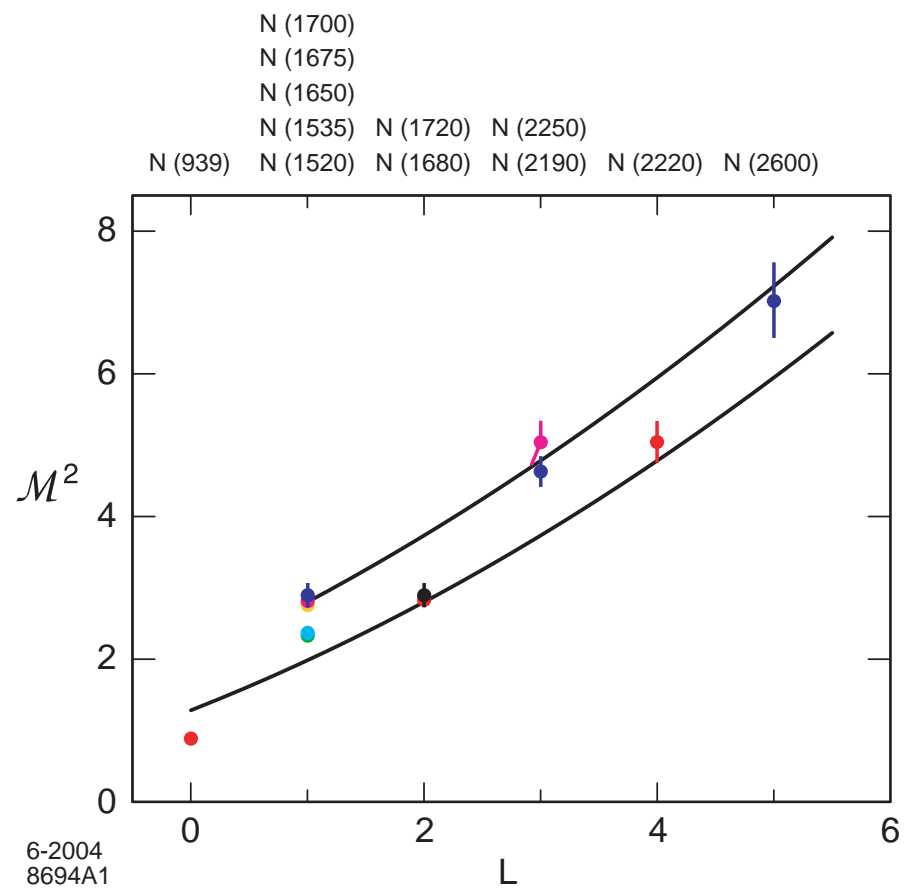

Figure 1: Nucleon orbital spectrum for a value of $\Lambda_{Q C D}=0.22 \mathrm{GeV}$. The lower curve corresponds to nucleon states dual to spin- $\frac{1}{2}$ string modes in the bulk. The upper curve corresponds to nucleon states dual to string- $\frac{3}{2}$ modes.

We present in Fig. 1 the orbital spectrum of the nucleon states and in Fig. 2 the $\Delta$ orbital resonances. We plot the values of $\mathcal{M}^{2}$ as a function of $L$. The nucleon states with intrinsic spin $S=\frac{1}{2}$ lie on a curve below the nucleons with $S=\frac{3}{2}$. We have chosen our boundary conditions by imposing the condition $\Psi^{+}\left(x, z=z_{o}\right)=0$ on the positive chirality modes for $S=\frac{1}{2}$ nucleons, and $\Psi_{\mu}^{-}\left(x, z=z_{o}\right)=0$ on the chirality minus strings for $S=\frac{3}{2}$. In contrast to the nucleons, all of the know $\Delta$ orbital states with $S=\frac{1}{2}$ and $S=\frac{3}{2}$ lie on the same trajectory. The boundary conditions in this case are imposed on the chirality minus string modes. The numerical solution corresponding to the roots of Bessel functions in (3), give the nonlinear trajectories indicated in the figures. All the curves correspond to the value $\Lambda_{Q C D}=0.22 \mathrm{GeV}$, which is the only actual parameter aside from the choice of the boundary conditions. The results for each trajectory show a clustering of states with the same orbital $L$, consistent with strongly suppressed spin-orbit forces; this is a severe problem for QCD models using one-gluon exchange. The results also indicate a parity degeneracy between states in the parallel trajectories shown in Fig. 1, as seen by displacing the upper curve by one unit of $L$ to the right, thus identifying parity doublets with the same total angular momentum [32]. Nucleon states with $S=\frac{3}{2}$ and $\Delta$ resonances fall on the same trajectory [28]. 


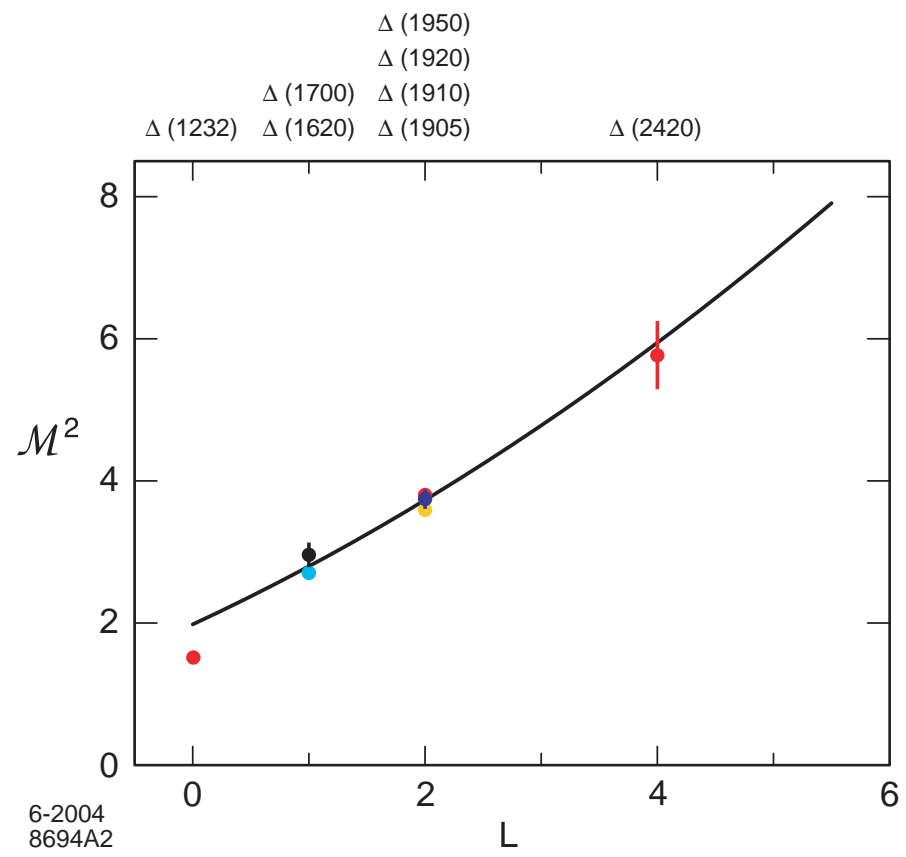

Figure 2: Delta orbital spectrum for $\Lambda_{Q C D}=0.22 \mathrm{GeV}$. The Delta states dual to spin- $\frac{1}{2}$ and spin- $\frac{3}{2}$ string modes in the bulk lie on the same trajectory.

In Fig. 3 we present the predictions for the $S^{5}, L=0$, baryon sector. The lowest states for $\kappa=0, L=0$, the $N(939)$ and the $\Delta(1232)$, are charged under $S^{5}$ and transform as a 4 under $S U(4)$. The $\kappa=1, L=0$ state, $|q q q \phi\rangle$, which transforms as a $\mathbf{2 0}$ under the internal $S U(4)$ symmetry of $S^{5}$, can be identified with the Roper $N(1440)$ and $\Delta(1600)$ resonances instead of radial excitations. The interpretation of the Roper is problematic in many models [27]. The $N(1710)$ can be identified with the $\kappa=2, L=0$ state, which transforms as $\mathbf{6 0}$ under $S U(4)$. We also predict a state with a mass around $1.85 \mathrm{GeV}$, not indicated in the figure, which corresponds to the first AdS radial mode.

\section{Concluding Remarks}

We have described aspects of the $\mathcal{N}=4$ SYM baryon spectrum introducing an open string sector and a scale in the far infrared AdS region. Since only one parameter, the QCD scale $\Lambda_{Q C D}$, is used, the agreement of the model with the pattern of the physical light baryon spectrum is remarkable. This agreement possibly reflects the fact that our analysis is based on a conformal template, which is a good initial approximation to QCD [25]. We have chosen a special representation to construct a three quark baryon, and the results are effectively independent of $N_{C}$. For glueballs [33] there is very little dependence on $N_{C}$ at small lattice spacing for $N_{C}>3$. The gauge/string 


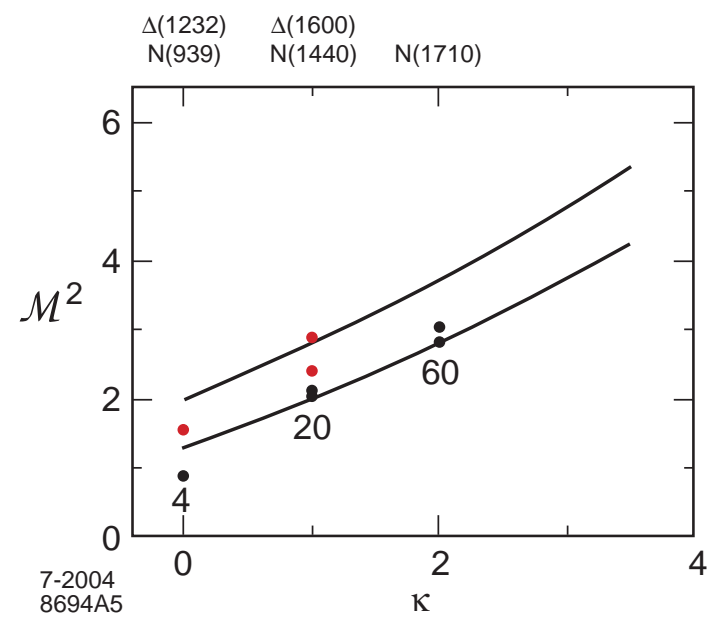

Figure 3: $S^{5}$ baryon spectrum sector for $L=0$ and $\Lambda_{Q C D}=0.22 \mathrm{GeV}$.

correspondence presented here appears as a powerful organizing principle to classify and predict the mass eigenvalues of all the known unflavored light baryons. It may also explain the Roper resonance $N(1440)$ and the $\Delta(1600)$, as baryon states containing a massless adjoint scalar gluon, dual to a string mode charged under $S^{5}$. The lowest states, the $N(939)$, and the $\Delta(1232)$ are more sensitive to the details of the metric near $z_{o}$, and a more elaborated model is possibly required to describe nonconformal aspects. A better understanding of the nature of the quantum fluctuations of the bulk geometry is also required. The results suggest that confinement properties and the structure of the QCD vacuum could depend on the nature of a higher dimensional dual gravity theory.

\section{Acknowledgements}

We thank Oleg Andreev, Simon Capstick, Lance Dixon, Jose Goity, Robert Jaffe, Igor Klebanov, David Mateos, David Richards, Jorge Russo and Matt Strassler for helpful comments.

\section{Added Note}

After completion of this work a mapping of meson operators to fluctuations of the supergravity background with orbifold projections has been conjectured in the framework of [22] for a three-dimensional boundary theory in the low energy limit of closed strings. See: J. Erdmenger and I. Kirsh, arXiv:hep-th/0408113. 


\section{References}

[1] J. M. Maldacena, Adv. Theor. Math. Phys. 2, 231 (1998) [Int. J. Theor. Phys. 38, 1113 (1999)] [arXiv:hep-th/9711200].

[2] S. S. Gubser, I. R. Klebanov and A. M. Polyakov, Phys. Lett. B 428, 105 (1998) [arXiv:hep-th/9802109].

[3] E. Witten, Adv. Theor. Math. Phys. 2, 253 (1998) [arXiv:hep-th/9802150].

[4] J. Polchinski and M. J. Strassler, Phys. Rev. Lett. 88, 031601 (2002) [arXiv:hepth/0109174].

[5] S. J. Brodsky and G. R. Farrar, Phys. Rev. Lett. 31, 1153 (1973); Phys. Rev. D 11, 1309 (1975).

[6] V. A. Matveev, R. M. Muradian and A. N. Tavkhelidze, Lett. Nuovo Cim. 7, 719 (1973).

[7] J. Polchinski and M. J. Strassler, JHEP 0305, 012 (2003) [arXiv:hep-th/0209211].

[8] S. J. Rey and J. T. Yee, Eur. Phys. J. C 22, 379 (2001) [arXiv:hep-th/9803001]; J. M. Maldacena, Phys. Rev. Lett. 80, 4859 (1998) [arXiv:hep-th/9803002].

[9] H. Boschi-Filho and N. R. Braga, Phys. Lett. B 560, 232 (2003) [arXiv:hepth/0207071]; arXiv:hep-th/0312231.

[10] R. C. Brower and C. I. Tan, Nucl. Phys. B 662, 393 (2003) [arXiv:hepth/0207144]; Nucl. Phys. Proc. Suppl. 119, 938 (2003).

[11] O. Andreev, Phys. Rev. D 67, 046001 (2003) [arXiv:hep-th/0209256]; Phys. Rev. D 70, 027901 (2004) [arXiv:hep-th/0402017].

[12] For a review see, O. Aharony, S. S. Gubser, J. M. Maldacena, H. Ooguri and Y. Oz, Phys. Rept. 323, 183 (2000) [arXiv:hep-th/9905111].

[13] S. J. Brodsky and G. F. de Téramond, Phys. Lett. B 582, 211 (2004) [arXiv:hepth/0310227].

[14] J. F. Gunion, S. J. Brodsky and R. Blankenbecler, Phys. Lett. B 39, 649 (1972); Phys. Rev. D8, 287 (1973).

[15] X. d. Ji, J. P. Ma and F. Yuan, Phys. Rev. Lett. 90, 241601 (2003) [arXiv:hep$\mathrm{ph} / 0301141]$.

[16] J. C. Plefka, Fortsch. Phys. 52, 264 (2004) [arXiv:hep-th/0307101]; D. Sadri and M. M. Sheikh-Jabbari, arXiv:hep-th/0310119. 
[17] F. Bigazzi, A. L. Cotrone, M. Petrini and A. Zaffaroni, Riv. Nuovo Cim. 25N12, 1 (2002) [arXiv:hep-th/0303191]. See also: J. M. Pons, J. G. Russo and P. Talavera, arXiv:hep-th/0406266; J. Russo, these proceedings.

[18] M. Henningson and K. Sfetsos, Phys. Lett. B 431, 63 (1998) [arXiv:hepth/9803251]; W. Muck and K. S. Viswanathan, Phys. Rev. D 58, 106006 (1998) [arXiv:hep-th/9805145].

[19] I. R. Klebanov and E. Witten, Nucl. Phys. B 556, 89 (1999) [arXiv:hepth/9905104].

[20] R. Camporesi and A. Higuchi, arXiv:gr-qc/9505009.

[21] D. J. Gross and H. Ooguri, Phys. Rev. D 58, 106002 (1998) [arXiv:hepth/9805129]; E. Witten, JHEP 9807, 006 (1998) [arXiv:hep-th/9805112].

[22] A. Karch and E. Katz, JHEP 0206, 043 (2002) [arXiv:hep-th/0205236].

[23] M. Kruczenski, D. Mateos, R. C. Myers and D. J. Winters, JHEP 0307, 049 (2003) [arXiv:hep-th/0304032]; D. Mateos, these proceedings.

[24] T. Sakai and J. Sonnenschein, JHEP 0309, 047 (2003) [arXiv:hep-th/0305049].

[25] S. J. Brodsky, arXiv:hep-ph/0408069.

[26] A. Volovich, JHEP 9809, 022 (1998) [arXiv:hep-th/9809009]; P. Matlock and K. S. Viswanathan, Phys. Rev. D 61, 026002 (2000) [arXiv:hep-th/9906077].

[27] S. Capstick and W. Roberts, Prog. Part. Nucl. Phys. 45, S241 (2000) [arXiv:nuclth/0008028].

[28] E. Klempt, arXiv:hep-ph/0404270.

[29] See for example, J. L. Goity, C. Schat and N. N. Scoccola, Phys. Lett. B 564, 83 (2003) [arXiv:hep-ph/0304167], and references therein. See also N. Matagne, these proceedings.

[30] LHPC Collaboration, to appear in the Proceedings of Lattice 2004; D. Richards, these proceedings.

[31] S. Eidelman et al. [Particle Data Group Collaboration], Phys. Lett. B 592, 1 (2004).

[32] L. Y. Glozman, Phys. Lett. B 475, 329 (2000) [arXiv:hep-ph/9908207].

[33] M. Teper, Nucl. Phys. Proc. Suppl. 109A, 134 (2002) [arXiv:hep-lat/0112019];

$\mathrm{M}$. Teper, these proceedings. 